\title{
Is our understanding of measurement evolving?
}

\author{
Luca Mari ${ }^{1}$ \\ ${ }^{1}$ Università Cattaneo - LIUC, C.so Matteotti, 22, 21053 Castellanza (VA), Italy
}

ABSTRACT

Traditionally understood as a quantitative empirical process, in the last decades measurement has been reconsidered in its aims, scope, and structure, so that the basic questions are again important: what kind of knowledge do we obtain from a measurement? what is the source of the acknowledged special efficacy of measurement? A preliminary analysis is proposed here from an evolutionary perspective.

\section{Section: RESEARCH PAPER}

Keywords: foundations of measurement; measurement and quantification; measurement as empirical process; measurement as representation

Citation: Luca Mari, Is our understanding of measurement evolving?, Acta IMEKO, vol. 10, no. 4, article 32, December 2021, identifier: IMEKO-ACTA10 (2021)-04-32

Section Editor: Francesco Lamonaca, University of Calabria, Italy

Received October 1, 2021; In final form November 20, 2021; Published December 2021

Copyright: This is an open-access article distributed under the terms of the Creative Commons Attribution 3.0 License, which permits unrestricted use, distribution, and reproduction in any medium, provided the original author and source are credited.

Corresponding author: Luca Mari, e-mail: Imari@liuc.it

\section{INTRODUCTION}

The terminology about and around measurement is often not so specific, and sometimes even a bit sloppy. For sure, a long tradition allows us to assume a reasonably common understanding of a phrase like "to measure the length (or the mass, or...) of a given physical body". But the claim that for example thermal comfort (as in [1]) is a measurable property is not as obviously meant in the same way by all relevant stakeholders. Do different experts refer to the same sort of situations when they talk about the measurement of thermal comfort? What do they mean when they use instead phrases like "determination of thermal comfort", "assessment of thermal comfort", "quantification of thermal comfort", "assignment of a value to thermal comfort"? And what are the conditions that make the determination, or the assessment, or... of thermal comfort a measurement?

Advancements of science and technology are not driven by terminological works, and therefore this kind of questions could be dismissed as immaterial if our goals are scientific or technological. Admittedly, indeed, clearer ideas about the meaning of a term - like "measurement", or "measurand", or "measurement uncertainty", and so on - do not improve our ability to design measuring instruments and perform measurements. Nevertheless, metrology (in the broad sense given by the International Vocabulary of Metrology (VIM: [2]): "science of measurement and its application", thus in principle not limiting it to physical quantities) is a very special body of knowledge, and this peculiarity suggests that terminology might be more important in metrology than in other experimental fields like physics and chemistry.

It is a fact that some key contents of metrology derive at least in part from social understanding and agreement, and not only from the outcomes of observation and experimentation. An obvious example is the identification of an individual quantity as the unit for a given kind, like the kilogram for mass: there can be empirical criteria to be taken into account, but the selection is ultimately conventional, and as such not falsifiable, in Popper's sense [3].

In fact, any discipline is grounded on some presuppositions and conventions that are chosen because they are mutually consistent, simple, elegant, ..., and not because they are true. The peculiarity of metrology is that this applies to a substantial part of its body of knowledge: even though measurement is an experimental process (the idea that a measurement can be a Gedankenexperiment, a thought experiment, sounds strange), it is as if the foundational role that metrology plays for all empirical sciences prevents it to obtain its own foundations somewhere else. Metrology is a foundation without a foundation [4].

While this seems to be a structurally obvious situation - if $X$ is founded on $X_{i-1}$ that is founded on $X_{i-2}$ that..., then the sequence must stop where an $X_{0}$ has no foundations -, acknowledging that metrology has sometimes the delicate role of $X_{0}$ could generate an embarrassing doubt: isn't metrology a "real" science then? And however, how can we forget that what is possibly the "most foundational" component of the metrology of the last 150 years is the Metre Convention, i.e., first of all a political treaty? Or as another example, closer to us in time, consider the interesting discussion about base quantities, 
triggered by the 2019 revision of the definitions of some units given in terms of numerical values assigned to some quantities modeled as constants (the speed of light in vacuum etc). Does this revision imply that the very idea of base quantity is now unjustified? Or that base quantities should be those of the defining constants (hence speed, action, etc)? Or finally that nothing should be changed on this matter (and therefore that length, mass, etc should remain the base quantities)? The question has in fact some importance, but no experiments can be designed to provide an answer: again, it is a matter of shared understanding of the pros and the cons, and then of agreement.

This solicits us to reconsider the role of terminology in metrology: where social agreement plays a key role, and when disagreements cannot be settled by seeking the truth, welldefined concepts and well-chosen terms - this is what terminological works aim at - may be useful, if not indispensable. Moreover, the fact that measurement is a fundamental enabler not only of top science, but also of technology, commerce, health care, environment protection, etc, adds a further reason to the special importance of terminology in metrology: again, this role requires shared concepts and terms, on which shared knowledge - like the one that guarantees the metrological traceability of measurement results - can be grounded. Metrology is a social body of knowledge.

Basically everything that has been considered so far applies also and particularly to the very starting point of metrology: what is measurement? how should 'measurement' be defined? (someone might object that the starting point of metrology is the definition of 'quantity', not the one of 'measurement', perhaps by referring to the fact that the first entry of the first chapter of the VIM is about 'quantity'; I respectfully disagree: 'quantity', like 'property', is a pre-metrological concept). In fact, there is nothing new in discussions about the scope of measurement and the terminological endeavor of providing an appropriate definition of 'measurement': the sometimes also harsh clashes about the measurability of psychological properties (i.e., the issue of whether psychometrics is actually about measurement) highlight that on the table there is way more than a dictionary issue. The distinction between, say, "my opinion on the competence of the candidate is..." and "the result of the measurement of the competence of the candidate is..." is not only about the occurrence of the term "measurement" in the second sentence, and in fact the position that psychological properties can be measured has been under scrutiny for decades [5]. Rather, a fundamental question is at stake here: what kind of knowledge do we obtain from a measurement? And also, given that "measurement is often considered a hallmark of the scientific enterprise and a privileged source of knowledge" [6]: "what [is] the source of [the] special efficacy" of measurement?" [7]. This is the subject to which the present paper is devoted.

\section{THE RECEIVED POSITION: MEASUREMENT AS A COIN WITH A EUCLIDEAN SIDE AND A GALILEAN SIDE}

It is remarkable that the answer to these questions is also historically ambiguous, with two main lines that have been developed [8].

On the one hand, the Euclidean tradition emphasizes the quantitative nature of measures, where in this sense "measurable" is basically synonym of "divisible by ratio", as clearly explained for example by De Morgan: “The term 'measure' is used [by Euclid] conversely to 'multiple'; hence [if] A and B have a common measure [they] are said to be commensurable" [9]. Hence, this concept of measure applies first of all to numbers: "a measure of a number is any number that divides it, without leaving a reminder. So, 2 is a measure of 4, of 8, etc" [10], as in fact stated by Euclid himself: "A number is part of a (nother) number, the lesser of the greater, when it measures the greater" (Euclid). There is nothing necessarily empirical in this concept of measure, and in fact "in the geometrical constructions employed in the Elements [...] empirical proofs by means of measurement are strictly forbidden" ([11]; in the introductory notes to the translation of Euclid's Elements).

On the other hand, the Galilean tradition emphasizes the empirical nature of measurement, where before Galileo "no one ever sought to get beyond the practical uses of number, weight, measure in the imprecision of everyday life" [12]. The tight connection between instrumentation and measurement witnesses the acknowledged role of measurement as a key enabler of the experimental method: a measurement is the process performed by making a physical device, i.e., a measuring instrument, interact with an empirical object according to the instructions provided by a measurement procedure.

Being quantitative and being empirical are orthogonal conditions: there are quantitative empirical processes, but a process may be quantitative and not empirical, or empirical and not quantitative. This means that in principle a process that is not a (Galilean) measurement might produce a (Euclidean) measure, and a process that is a (Galilean) measurement might not produce a (Euclidean) measure (such a lexical peculiarity was already highlighted by Bunge [13], who discussed the difference between 'measure' and 'measurement'; this becomes further clear by comparing the scopes of measure theory and a measurement theory).

However, historically Galileo came later, and drew from Euclid and his interpretations, so that the principled independence became a factual convergence: (Galilean) measurement was assumed to be a quantitative process, that produces (Euclidean) measures. The lexicon maintains some traces of the coexistence of these two standpoints and of the interest of endorsing both, as witnessed in particular by the expression "weights and measures", as if weighing were not a way to measure. Indeed, Euclid was concerned with geometric quantities, so that in the Euclidean tradition measurable (or: "mensurable", as it was said) were considered geometric quantities: according to Hutton, "mensuration" is "the act, or art, of measuring figured extensions and bodies, or of finding the dimensions and contents of bodies, both superficial and solid" [10], so that for example in the case of temperature he used the term "observation" (this shows that the scope of measurement already broadened in the past!).

Plausibly, the synthesis of the Euclidean and the Galilean standpoints led to the idea that only extensive (taken from Euclid) physical (taken from Galileo) quantities are measurable, at least in the fundamental sense specified by Campbell [14]. With some simplification, we may then summarize that the received view about 100 years ago was about measurement as characterized by two complementary components: measurement as a coin with a Euclidean side and a Galilean side.

Such a position is so strict - it is the outcome of the intersection of two independent standpoints, and thus inherits two sets of constraints - that not surprisingly trying to overcome it has been a target for several decades. In this perspective, the well known report of the Ferguson committee to the British Association for the Advancement of Science, published in 1940 [15], stating that "the main point against the measurability of the 
intensity of a sensation was the impossibility of satisfactorily defining an addition operation for it" [16], can be read as a move to defend the orthodoxy of the synthesis of the Euclidean and the Galilean sides of the coin.

\section{RETHINKING THE RECEIVED POSITION}

In the last century the assumptions that measurement is quantification (the Euclidean side) and is about (geometric) physical properties only (the Galilean side) have been reanalysed, apparently by asking if really both such requirements need to be fulfilled, and to what extent. In particular, from Stevens' theory of types of scales [17] representationalism [18] explored how to broaden the Euclidean side, sometimes by simply dropping any reference to the Galilean side. In this sense we can read statements claiming that "the theory of measurement is difficult enough without bringing in the theory of making measurements" [19], or that a representation theorem "makes the theory of finite weak orderings a theory of measurement, because of its numerical representation" [20].

In this complex context, the definition given by the VIM "process of experimentally obtaining one or more quantity values that can reasonably be attributed to a quantity" [2] - is still quite conservative, with both the Euclidean side ("quantity") and the Galilean side ("experimental") explicitly maintained. Is it sufficient for our society, that requires criteria of trust about determining / assessing / attributing values to ... properties, like thermal comfort, that are not necessarily quantitative and are not entirely empirical? And is it sufficient for our society, in which the widespread digitalization is producing larger and larger amounts of data (the so-called "big data" phenomenon)? Indeed, in an age of fake news and post-truth, providing criteria that make explicit and possibly operational the VIM condition of "reasonable attribution", and therefore such that not any data deserve to be called "measurement results", seems to be a valuable achievement.

In other words, our complex society would definitely benefit from an effective answer to Kuhn's question about the source of the special efficacy of measurement, while at the same time pushing toward reconsidering the actual necessity of Euclidean and Galilean conditions. And in fact conservative positions like VIM's are challenged today, so that measurement seems to have become a moving target. A significant and authoritative example is the standpoint of the US National Institute of Standards and Technology's Simple guide for evaluating and expressing the uncertainty of NIST measurement results, that defines 'measurement' as "an experimental or computational process that, by comparison with a standard, produces an estimate of the true value of a property of a material or virtual object or collection of objects, or of a process, event, or series of events, together with an evaluation of the uncertainty associated with that estimate, and intended for use in support of decision-making" [21]. With a mix of tradition (the reference to true values) and innovation (the evaluation of uncertainty as a necessary condition), here both Euclidean and Galilean conditions have been dropped: also non-quantitative properties are in principle measurable, and measurement can be also a non-empirical process about non-empirical ("virtual") objects.

Is our understanding of what measurement is still evolving then, and in which direction(s)?

\section{SOME POSSIBLE EVOLUTIONARY PERSPECTIVES}

Listing some necessary conditions that characterize measurement, and that plausibly are generally accepted, is not a hard task: measurement is (i) a process (ii) designed on purpose, (iii) whose input is a property of an object, and (iv) that produces information in the form of values of that property. Indeed, (i) removes the ambiguity of using the term "measurements" also for the results of the process; (ii) highlights that measurement is not a natural process "that happens"; (iii) establishes that phrases like "to measure an object" are not correct, because measured are properties of objects, and not objects as such; (iv) characterizes measurement as an information process. However, not any such process is a measurement, thus acknowledging that not any data acquisition is a measurement. We may call "property evaluation" a process fulfilling (i)-(iv). What sufficient conditions characterize measurement as a specific kind of property evaluation? The answer does not seem as easy.

The term "measurement" does not have a single inherent meaning and is not trademarked, so that nobody can be prevented from using it as she/he likes. Nevertheless, without a common foundation, a foundational body of knowledge, as metrology is, is at risk of emptying, or at least of becoming unable to provide a convincing, socially agreeable, and useful answer to Kuhn's question: indeed, not any data acquisition process is claimed to have a "special efficacy".

As discussed above, the traditional, i.e., Euclidean \& Galilean, answer to this question relies on coupling quantification and instrumentation: the assumption that only quantitative properties are measurable guarantees that measurement results are embedded in the nomological network generated by physical laws, from which specific, and then falsifiable, predictions and inferences can be drawn and the hypothesis of the correctness of the measurement results can be corroborated in turn; and the requirement that measuring instruments are empirical devices guarantees that the degree of objectivity of measurement results can be assessed by analysing how such instruments behave. This is the safe starting point. But if both such sides are erased, what remains of the coin?

While sticking to the tradition is safe, it might be too strict with respect to what our society needs, as the definition of 'measurement' given by the NIST seems to suggest. This is a key challenge for metrology, whose solution is then a matter of social responsibility, not truth seeking.

In this context, in which respect of the tradition and new societal needs must be balanced, the possible evolutionary perspectives of measurement can be considered along four main complementary, though somewhat mutually connected, dimensions:

- measurable entities as quantitative or non-quantitative properties (i.e., the reference to the Euclidean tradition);

- measurable entities as physical or non-physical properties;

- measuring instruments as technological devices or human beings;

- measurement as an empirical or an informational process, and therefore the relation between measurement and computation (i.e., the reference to the Galilean tradition).

Again with the explicit admission that at stake here is adequacy, not truth, let us shortly discuss each of these issues.

\subsection{Measurable entities as quantitative or non-quantitative properties}

As Stevens' theory of scale types shows, the distinction between quantitative and non-quantitative properties is not 
binary. The strongest type is absolute: an absolute evaluation is additive and has both a natural unit and a natural zero, as is the case of counting. The weakest type is nominal: a nominal evaluation only classifies objects by property. Several intermediate types exist between absolute and nominal (e.g., ratio, interval, and ordinal, in the initial version of Stevens' theory), and there is not a single objective criterion to decide where a property stops to be quantitative and becomes nonquantitative. For example, according to the VIM a total order is sufficient for a property to be a quantity, whereas the axiomatic approaches developed from Holder's [22] consider "continuity as a feature of the scientific concept of quantity". The connection between being quantitative and being measurable inherits this ambiguity [23]. For sure, evaluations performed in richer scales convey more structural information, but this does not seem a sufficient criterion to rule out any given type from the scope of measurement: only by convention the decision can be made whether nominal (or ordinal, or...) evaluations can be measurements (and what conditions are required to make quantitative a property, for what it is worth).

\subsection{Measurable entities as physical or non-physical properties}

Independently of the scale type, the condition of being measurable could be connected to the nature of the considered properties, and in particular to their being physical. A plausible, good justification is the requirement to assess, and possibly to control, the degree of objectivity of the behaviour of the measuring instrument. Indeed, this is guaranteed by the operation of a physical sensor at the core of the instrument, where only a physical (or chemical, or biological) property may affect the transduction performed by the sensor. However, a non-physical property, like thermal comfort, may be evaluated as a function of one or more physical properties, such as air temperature and humidity, in a process that is structurally the same as those traditionally considered to be indirect measurements, and where values of such physical properties can be then obtained by means of sensors. The key difference between the evaluation of, say, thermal comfort and density the latter being a case of indirect measurement through the measurement of mass and volume - is that non-physical properties miss the rich nomological network provided by physics, so that their combination function is not as substantially validated. Whether this is sufficient to rule out non-physical properties from the scope of measurement seems to be again a matter of convention.

\subsection{Measuring instruments as technological devices or human beings}

Complementary to the option that also non-physical properties are measurable, some evaluations directly performed by human beings could be accepted as measurements. The relatively long history of what has been considered psychophysical measurement shows that there is nothing really new in this. There are in fact three strategies to develop humanbased instruments that attribute values to (physical or, more usually, non-physical) properties. First, the behaviour of a "typical" individual is idealized in a model, like in the case of the luminosity function, that describes the sensitivity of a "standard" human eye to different wavelengths. Second, a statistic of the behaviour of a set of human beings (e.g., their average response) is considered, under the assumption that individual peculiarities are compensated in a sufficiently large sample, like when the quality of a product or a service is evaluated by synthesizing the responses given by several customers. Third, an individual or a small group of individuals operates, under the condition that they are domain experts and therefore their evaluation can be considered to be calibrated against some agreed standards, like in the case of gymnastics judges and wine sommeliers. While at least some cases of the first strategy are widely accepted as measurements, as the inclusion of the candela as the SI unit of luminous intensity witnesses, whether and under what conditions human beings can be measuring instruments, possibly operating with the support of guidelines, checklists, etc, is again a controversial issue.

\subsection{Measurement as an empirical or an informational process}

Measurements are aimed at attributing values to properties: since values are information entities, any measurement must then include an informational component. Rather, the issue here is whether there can be measurements that are entirely informational processes, with no empirical components at all (note that this is not the case of gymnastics judges and wine sommeliers mentioned above: they are expected to operate by (empirically) observing gym competitions and tasting wines). There are at least two cases at stake. One is about the evaluation of properties that are in turn informational, for example the number of lines of code in the source of a software program. As quoted in section 3, the "computational process" about a "virtual object" to which the NIST definition refers could be such, and actually shares several structural features with the processes that are commonly accepted to be measurements. More controversial is instead the hypothesis to consider to be a measurement any computation performed on values of properties, like when one is asked to compute the acceleration that a given force would produce if applied to a body of a given mass. Of course, the information on the force and the mass (the "input quantities") could also include an uncertainty, that in this case should be somehow propagated to the acceleration, and someone could decide that this is sufficient to make such a computation a measurement, by then accepting that what has been propagated is a "measurement" uncertainty, through a "measurement" model. In an evolutionary situation, this is also a possible standpoint.

\section{CONCLUSIONS}

Were we in charge of updating a definition of 'measurement', for example for a new edition of the VIM, what would we propose, then? How tightly would we stick to the traditional conception of measurement as a quantitative empirical process? Or what criterion would we adopt toward a different, and plausibly broader, characterization?

Conscious that there is not one right, or true, answer, and by taking for granted the necessary conditions listed at the beginning of section 4, I dare to suggest - as a working hypothesis - that the most fundamental and most characterizing task of measurement is to produce information that is explicitly and socially justifiable (it is also the conclusion reached in [8]). This is not related to the quality of the produced information nor to the scope of the process (as the VIM states, measurement should be characterized "irrespective of the level of measurement uncertainty and irrespective of the field of application" [2]), but to the condition that a measurement is a "white box" process, so that the quality of its results - be it good or bad - can always in principle be justified. Accordingly, the source of the special efficacy of measurement, as investigated by Kuhn, is the possibility to reach a common understanding on how 
trustworthy its results are, along the two key dimensions of the objectivity and the intersubjectivity of the provided information [24], [25]. This explains the strategic importance of some components of the metrology of physical quantities, like the widely agreed definition of measurement units and the condition of metrological traceability of measurement results to such units through the appropriate calibration of measuring instruments. Whether and how a sufficient objectivity and intersubjectivity of the information produced by processes that aim at being acknowledged as measurements can be obtained: in the perspective we have proposed here, this is the key challenge for an evolutionary understanding of measurement.

\section{REFERENCES}

[1] ISO 7730:2005, Ergonomics of the thermal environment Analytical determination and interpretation of thermal comfort using calculation of the PMV and PPD indices and local thermal comfort criteria, International Organization for Standardization, Geneva, 2005.

[2] JCGM 200:2012, International Vocabulary of Metrology - Basic and general concepts and associated terms, 3rd ed., Paris: Joint Committee for Guides in Metrology, 2012. Online [Accessed 15 December 2021] https://www.bipm.org/en/committees/jc/jcgm/publications.

[3] K. Popper, The logic of scientific discovery, Routledge, Abingdon-on-Thames, 1959.

[4] L. Mari, The problem of foundations of measurement, Measurement, 38(4) (2005) pp. 259-266. DOI: $10.1016 /$ j.measurement..2005.09.006

[5] J. Michell, Measurement in psychology: A critical history of a methodological concept, Cambridge University Press, Cambridge, 1999.

[6] E. Tal, Measurement in science, in: The Stanford Encyclopedia of Philosophy, E.N. Zalta (ed.), 2020. Online [Accessed 15 December 2021]

https://plato.stanford.edu/archives/fall2020/entries/measurem ent-science

[7] T.S. Kuhn, The function of measurement in modern physical science, Isis, 52(2) (1961) pp. 161-193.

[8] L. Mari, M. Wilson, A. Maul, Measurement across the sciences Developing a shared concept system for measurement, Springer Nature, 2021. Online [Accessed 15 December 2021] https://link.springer.com/book/10.1007\%2F978-3-030-65558-7

[9] A. De Morgan, The connection of number and magnitude: An attempt to explain the fifth book of Euclid, Taylor and Walton, London, 1836. Online [Accessed 15 December 2021] https://archive.org/details/connexionofnumbe00demorich

[10] C. Hutton, A mathematical and philosophical dictionary, Johnson, London (freely available on Google books), 1795.

[11] Euclid's Elements of geometry, the Greek text of J. L. Heiberg (1883-1885) edited, and provided with a modern English translation, by Richard Fitzpatrick, 2008. Online [Accessed 15 December 2021] http://farside.ph.utexas.edu/Books/Euclid/Euclid.html

[12] A. Koyré, Du monde de l'à peu près à l'univers de la précision, in: A. Koyré (Ed.), Etudes d'histoire de la pensée philosophique (pp. 341-362), Gallimard, Paris, 1948.

[13] M. Bunge, On confusing 'measure' with 'measurement' in the methodology of behavioral science, in: M. Bunge (Ed.), The Methodological Unity of Science, D. Reidel, Dordrecht-Holland, 1973.

[14] N. R. Campbell, Physics: the elements, Cambridge University Press, Cambridge, 1920.

[15] A. Ferguson, C. S. Myers, R. J. Bartlett, H. Banister, F. C. Bartlett, W. Brown, W. S. Tucker, Final report of the committee appointed to consider and report upon the possibility of quantitative estimates of sensory events. Report of the British Association for the Advancement of Science, 2 (1940) pp. 331-349.

[16] G. B. Rossi, Measurability, Measurement, 40 (2007) pp. 545-562. DOI: $10.1016 /$ j.measurement.2007.02.003

[17] S. S. Stevens, On the theory of scales of measurement, Science, 103(2684) (1946) pp. 677-680.

[18] D. H. Krantz, R. D. Luce, P. Suppes, A. Tversky, Foundations of measurement, Vol. 1: Additive and polynomial representations, Academic Press, New York, 1971.

[19] H.E. Kyburg, Theory and measurement, Cambridge University Press, Cambridge, 1984.

[20] P. Suppes, Representation and invariance of scientific structures, CSLI Publications, Stanford, 2002.

[21] A. Possolo, Simple guide for evaluating and expressing the uncertainty of NIST measurement results, Technical Note, National Institute of Standards and Technology, Gaithersburg, MD, 2015.

DOI: $10.6028 /$ NIST.TN.1900

[22] O. Holder, Die Axiome der Quantität und die Lehre vom Mass. Berichte über die Verhandlungen der Koeniglich Sächsischen Gesellschaft der Wissenschaften zu Leipzig, MathematischPhysikaliche Klasse, 53 (1901) pp. 1-46. Part 1 translated in J. Michell, C. Ernst, The axioms of quantity and the theory of measurement, J. Mathematical Psychology, 40(3) (1996) pp. 235252.

DOI: $\underline{10.1006 / \mathrm{imps} .1997 .1178}$

[23] L. Mari, A. Maul, D. Torres Irribarra, M. Wilson, Quantities, quantification and the necessary and sufficient conditions for measurement, Measurement, 100 (2016) pp. 115-121. DOI: $10.1016 /$ j.measurement.2016.12.050

[24] A. Maul, L. Mari, D. Torres Irribarra, M. Wilson, The quality of measurement results in terms of the structural features of the measurement process, Measurement, 116 (2018) pp. 611-620. DOI: $10.1016 /$ j.measurement.2017.08.046

[25] A. Maul, L. Mari, M. Wilson, Intersubjectivity of measurement across the sciences, Measurement, 131 (2019) pp. 764-770. DOI: $\underline{10.1016 / \text { j.measurement..2018.08.068 }}$ 